\title{
Cellular automaton for bacterial towers
}

\author{
J. O. Indekeu* and C. V. Giuraniuc \\ Laboratorium voor Vaste-Stoffysica en Magnetisme, \\ Celestijnenlaan 200 D, Katholieke Universiteit Leuven, \\ B-3001 Leuven, Belgium
}

\begin{abstract}
A simulation approach to the stochastic growth of bacterial towers is presented, in which a non-uniform and finite nutrient supply essentially determines the emerging structure through elementary chemotaxis. The method is based on cellular automata and we use simple, microscopic, local rules for bacterial division in nutrient-rich surroundings. Stochastic nutrient diffusion, while not crucial to the dynamics of the total population, is influential in determining the porosity of the bacterial tower and the roughness of its surface. As the bacteria run out of food, we observe an exponentially rapid saturation to a carrying capacity distribution, similar in many respects to that found in a recently proposed phenomenological hierarchical population model, which uses heuristic parameters and macroscopic rules. Complementary to that phenomenological model, the simulation aims at giving more microscopic insight into the possible mechanisms for one of the recently much studied bacterial morphotypes, known as "towering biofilm", observed experimentally using confocal laser microscopy. A simulation suggesting a mechanism for biofilm resistance to antibiotics is also shown.
\end{abstract}

Key words: bacteria, growth, biofilm, fractal PACS: 87.18.La

\section{Introduction}

Bacteria can either grow freely or adhere to surfaces. In the latter case one obtains structures ranging from compact colonies with self-affine surfaces, over diffusion-limited self-similar aggregates, to towering-pillar or mushroom

\footnotetext{
* Corresponding author

Email addresses: joseph.indekeu@fys.kuleuven.ac.be (J. O. Indekeu), claudiu.giuraniuc@fys.kuleuven.ac.be (C. V. Giuraniuc).
} 
shaped biofilms [1]. Bacterial biofilms are commonly present in daily life; they typically flourish on substrates in aqueous environments, from sewage pipes to human teeth. In addition to performing physical measurements, it is necessary to introduce theoretical models for biofilm development. These models can be used in the prediction and/or understanding of the system's evolution in experiment or simulation and they can also improve the understanding of intrinsic features. As an example, the efficiency of an antibiotic can depend on the spatial distribution and organization of bacteria.

Two different approaches have been developed over time: they use either microscopic or macroscopic rules. While macroscopic models can give more easily a global image of the biofilm, the microscopic ones describe better the behaviour at the level of the smallest biofilm unit - the "cell". Ideally suited as elemental units of a cellular automaton approach, these cells contain typically a very small number of bacteria, or lots of nutrient particles, or simply consist of water-filled compartments. Biophysical properties are not imposed a priori but emerge as a result of local microscopic rules [1].

One of the best known theoretical biology models for population growth, which has since long been applied in various other fields, is the Eden Model [2]. Various features have been added to this model, in order to describe different types of situations that appear in specific cases. Other approaches are diffusion limited aggregation (DLA)[3] and, more generally, the cellular automata methods [4]. In this article we shall present a method based on this last technique, which amounts to nutrient-limited aggregation in which chemotaxis is the driving mechanism and diffusion plays a minor role.

\section{Model description}

The general framework we employ is the cellular automata (CA) method. CA are discrete dynamical systems whose behavior is completely specified in terms of a local relation (rules). CA can be thought of as a stylized universe. Space is represented by a uniform grid, with each site or "cell" containing a few bits of data. Time advances in discrete steps, and the laws of the universe are expressed by a simple recipe - say, a small look-up table - through which at each step each cell computes its new state from that of its close neighbors. The system's laws are local and uniform.

For our purpose the environment is a three-dimensional simple cubic lattice of size $(l \times w \times h=81 \times 81 \times 27)$. Each lattice site is in one of a finite number of distinct states. Usually the number of states is small but, in principle, any finite CA model over a finite alphabet can be defined. The state of a cell (site) changes according to some rules. There are two distinct classes 
of automata: synchronous, which allows for simultaneous update of all cells' states, and asynchronous. The updating order can be deterministic or random in the asynchronous class. The local rules of a certain CA are essentially determined by the considered neighborhood, consisting of the 6 nearest neighbors, 12 next-nearest and 8 next-next-nearest neighbors of a given site in the cubic lattice, 26 in total. We have used asynchronous updating.

We start with a homogeneous substrate layer of bacteria of size $81 \times 81 \times 10$ submerged in water. The water initially occupies a $81 \times 81 \times 17$ volume. We deposit at random large nutrient chunks on the substrate, conveniently approximated in shape by compact rectangular pillars of cells, of size $27 \times 27 \times 10$. The pillars are deposited with probability $P$ over a grid of 9 macroscopic square substrate plaquettes of area $27 \times 27$, each containing many substrate cells. This process creates a strongly non-uniform nutrient supply partly covering the substrate. Bacterial cells may multiply provided they eat neighboring nutrient cells (nutrient is then replaced with bacteria). This process is characterized by a growth probability $G$ and represents binary division coupled to elementary chemotaxis, as the bacteria grow and spread preferentially in the direction of highest nutrient concentration. Clearly, the greater the number of neighboring cells occupied by nutrient, the greater is the probability that a bacterial cell will divide. For the time being, we disallow diffusion, and leave an examination of nutrient diffusion effects to a later section.

Technically, we choose at random a pair of neighboring cells and if the pair consists of nutrient and bacteria we replace the nutrient by bacteria with probability $G$ and then pass on to another randomly chosen pair. We take measurements at time steps of $(l \times w \times h)$ updates, so that each cell has on average been visited once in every time step, and record bacterial population and nutrient configuration.

\section{$3 \quad$ Results}

\subsection{Nutrient-limited growth without diffusion}

We assume a probability $P$ for putting at random nutrient pillars over the coarse-grained substrate grid and a probability $G$ for bacterial cells to consume adjacent nutrient cells and subsequently divide, thereby occupying also the nutrient cell. As can be seen in the vertical section shown in Figure 1, the bacteria develop towers in the places where the nutrient was deposited at random. Note the developing roughness of the originally flat bacteria/nutrient interface. Within a tower the growth process is Eden-like (in three dimensions), since all perimeter cells carry nutrient. Eventually, however, the bacteria fill 
up the towers and perfectly flat walls and rooftops result.

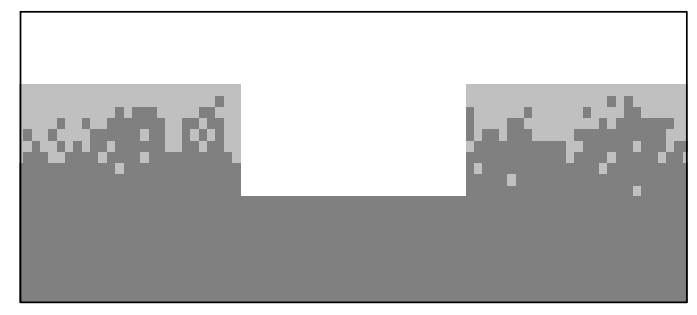

Fig. 1. Vertical section $(81 \times 27)$ through the cellular lattice system of bacteria (dark), nutrient (light) and water (white), after 20 time steps. The nutrient deposition probability $P$ equals 0.33 and the bacterial growth probability $G$ equals 0.2 . Two bacterial towers grow where nutrient pillars were placed. Eden-like growth takes place inside the pillars until eventually they are filled compactly with bacteria. Note that bacterial cells which seem detached from the colony in this $2 \mathrm{D}$ section, are connected in the $3 \mathrm{D}$ structure.

Let us denote by $N(t)$ the bacterial population, in units of cells, in excess of the initially present substrate population, so that $N(0)=0$. Plots of $N(t)$, measured every time step, reveal a characteristic sigmoid shape, common for various biological growth processes approaching a finite carrying capacity [5]. Since the deposition of nutrient is stochastic, the carrying capacity $K$ depends on the sample, and consequently a $K$-distribution arises, each $K$ being simply equivalent to the total mass of deposited nutrient pillars. The speed of saturation of the population towards $K$ is, of course, faster when $G$ is bigger and for each population curve we can discern three distinct regimes as illustrated in Figure 2, for which we took $P=0.33$.

i) The initial growth is not exponential - it would be exponential in free binary division - but is limited by the amount of nutrient in the first horizontal layer adjacent to and in contact with the bacterial substrate. The population increase is just proportional to the contact area $A$ and to $G$, leading to a linear initial growth. This stage can be characterized by a linear saturation time $\tau_{\text {lin,sat }}$, proportional to $1 / G$. This is the average time needed to build a bacterial tower in the absence of interface fluctuations (cf. "mean-field" approximation), and is found by simple linear extrapolation of the initial growth. ii) The growth soon accelerates as the area of the bacteria/nutrient contact boundary increases by interface roughening and the appearance of nutrient "holes" (see Fig.1). The roughness, caused by height fluctuations, is limited by the finite horizontal width of the nutrient pillar(s), resulting in a saturating contact area, again implying a linear growth regime. iii) This regime in turn crosses over to an exponentially fast convergence of the growth towards $K$, which is the asymptotic behaviour for long times. Indeed, the bacteria reaching the ceiling of the nutrient pillar fall short of nutrient and eventually all nutrient cells are consumed. The sigmoid shape arises from the succession of 

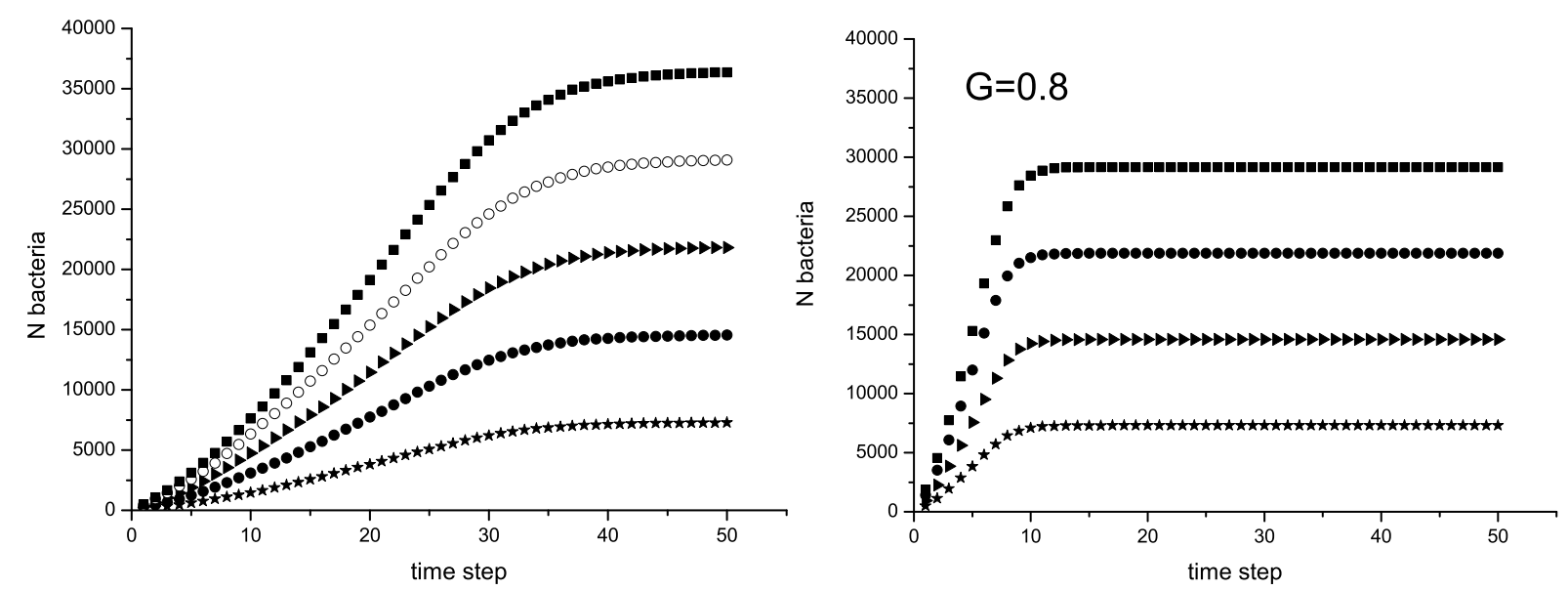

Fig. 2. The number of bacterial cells versus time for growth probability $G=0.2$ (left) and $G=0.8$ (right). The different curves, each with its own carrying capacity $N(\infty)=K$, correspond to different numbers of randomly deposited nutrient pillars. The fluctuations around a given curve are small, so that each curve accurately represents a narrow band of very similar curves. For $G=0.2$ the linear saturation time $\tau_{\text {lin,sat }}$ equals about 65 time steps, for all curves, and for $G=0.8$ about 12 time steps, for all curves. The growth of $N(t)$ first accelerates and then comes to a halt, leading to a characteristic sigmoid shape.

these three regimes i)-iii). The dependence of the bacterial population on time can be understood most clearly from the time evolution of the area $A(t)$ of the bacteria/nutrient contact interface, shown in Figure 3.

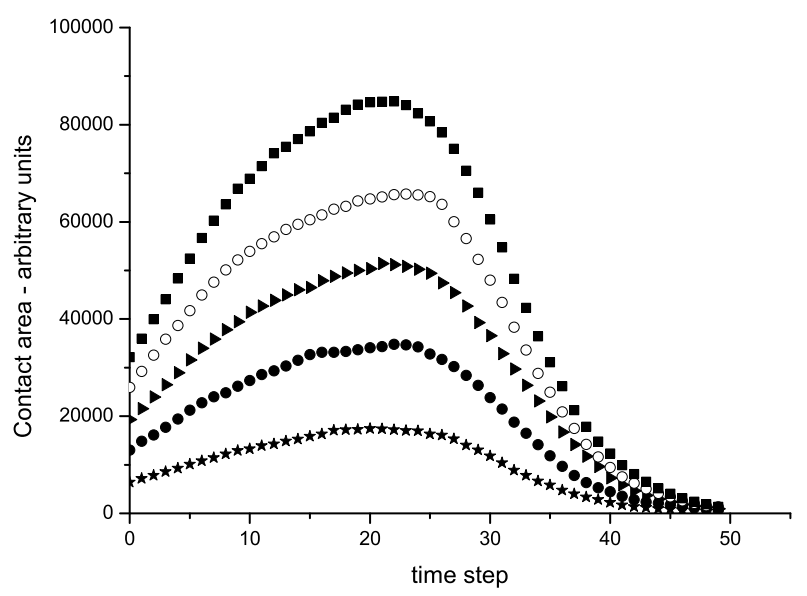

Fig. 3. Contact area of the bacteria/nutrient interface in units of pairs of neighboring cells consisting of adjacent bacteria and nutrient. The nutrient deposition probability is $P=0.33$ and the growth probability $G=0.2$. The curves shown are proportional to the time derivative of the population curves in Fig.2 (left). Note the finite constant for initial times, the maximum for intermediate times and the exponential decay for long times. 
Actually, we have

$$
\frac{d N(t)}{d t} \propto G A(t)
$$

Asymptotically for long times, the decrease of the nutrient mass, $M(t)$, which equals the increase of the bacterial population $N(t)$, is simply proportional to the number of nutrient cells, since these become isolated, whence independent, and surrounded by neighboring bacterial cells. Thus we have

$$
d M \propto-M G d t
$$

It follows that the asymptotic behavior of $N$ for long times can be written as

$$
N(t) \sim K\left(1-e^{-\left(t-t_{0}\right) / \tau}\right)
$$

where the saturation time $\tau$ is proportional to $1 / G$ and depends only weakly on the probability $P$ of depositing nutrient pillars, because these can to a first approximation (especially for small $P$ ) be considered as "non-interacting". Numerically, $1 / \tau \approx 0.85 \pm 0.1$ for $G=0.8$, implying a very short time scale for the actual exponential saturation in Fig.2 (right) compared to the linear saturation time. The time $t_{0}$ denotes the moment for which a zero excess population, $N\left(t_{0}\right) \equiv 0$, would result by extrapolating backwards the asymptotic behavior. This time equals about $t_{0}=16$ for all curves with $G=0.2$ and about $t_{0}=5$ for all curves with $G=0.8$ (Fig.2). It is remarkable that the three characteristic times $\tau_{\text {lin,sat }}, \tau$ and $t_{0}$ are, within the accuracy of our computations, independent of the carrying capacity $K$.

\subsection{Nutrient-limited growth with nutrient diffusion}

We have performed simulations similar to the ones described above, but in which we have included stochastic nutrient diffusion. A probability $I$ is introduced for the interchange of adjacent cells, one occupied with nutrient and the other with water. This model is closer to physical, or biological, reality since the nutrient in general is soluble in water. In our simulations diffusion turns out to be necessary for generating the observed porous morphologies with fractal-like surface roughness and possible overhangs leading to mushroomshaped towers, also seen experimentally [1]. Figures 4 and 5 show relevant vertical and horizontal sections through the developing biofilm structure.

In the presence of nutrient diffusion, bacterial reproduction generates a two-

step biofilm growth. Firstly, Eden-like growth driven by chemotaxis takes 

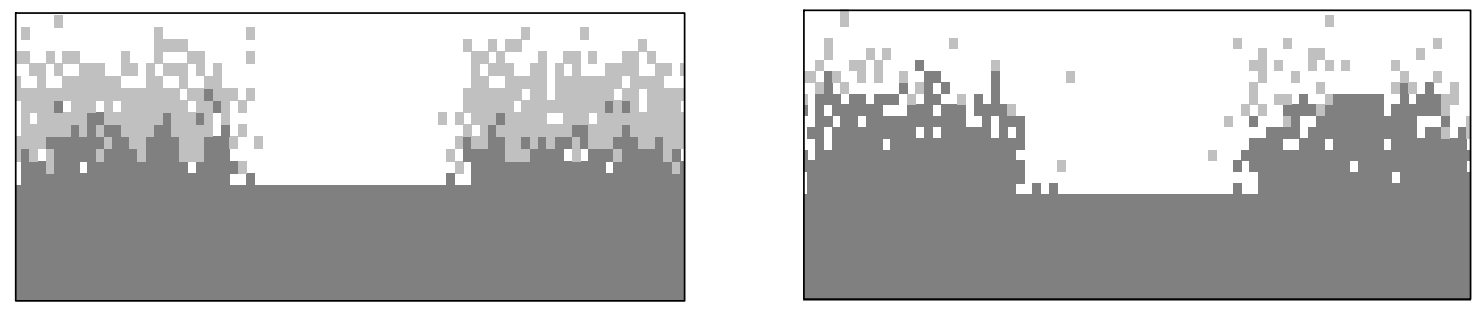

Fig. 4. Vertical sections through the biofilm at an early time $t=6$ (left) and at a later time $t=20$ (right), for $P=0.33, G=0.6$ and diffusion probability $I=0.6$. A cross-over from Eden to DLA growth occurs by the time most of the nutrient pillars is consumed.

place in the nutrient pillars (as for zero diffusion). Secondly, DLA-type of growth roughens and ramifies the bacterial towers as the wandering nutrient recombines by aggregation. Clearly, the long time DLA process determines the biofilm morphotype. A remarkable property of the fractal character of the emerging structure becomes detectable in Fig.5 (horizontal slices through the biofilm). Sections through the top layers reveal a cascade or hierarchy of length scales. Water "lakes" in bacterial "land" develop into bacterial "islands" surrounded by water, over a height difference of just a few layers. This is strikingly similar to the structure that is inherent in the hierarchical population model, where it is imposed ad hoc. In contrast, here in the simulations it occurs spontaneously.

In Figure 6 we observe once more the basic sigmoid shape of the $N(t)$-curves describing the time evolution of the bacterial population, for 10 samples. Thus the shape of the population curves is robust with respect to the new perturbation; it is not much influenced by diffusion, at least not for short and medium times. The new feature brought about by diffusion is the existence of a long tail in $N(t)$, beginning after the formation of bacterial towers and ending when all detached nutrient has diffused "back" towards, and is eaten by, the bacterial colonies. This tail is still exponential, but with a decay time roughly 100 times longer than in the absence of diffusion. For example, for $P=0.33$ and $G=0.8$, we find $1 / \tau=0.009 \pm 0.001$. Interestingly, this saturation rate turns out to be independent of $I$ provided $I>0$, at least to the accuracy of our computations so far (averaging over samples, and for $I=0.2,0.4,0.6,0.8$ and 1). One may speculate that this could have been anticipated qualitatively, since weak diffusion leads to minor detachment of nutrient and swift recombination. On the other hand, strong diffusion entails more detachment but also greater mobility, whence also swift aggregation. The DLA process' efficiency seems largely independent of the diffusion probability.

The exponential character of the saturation can be understood as follows. For 

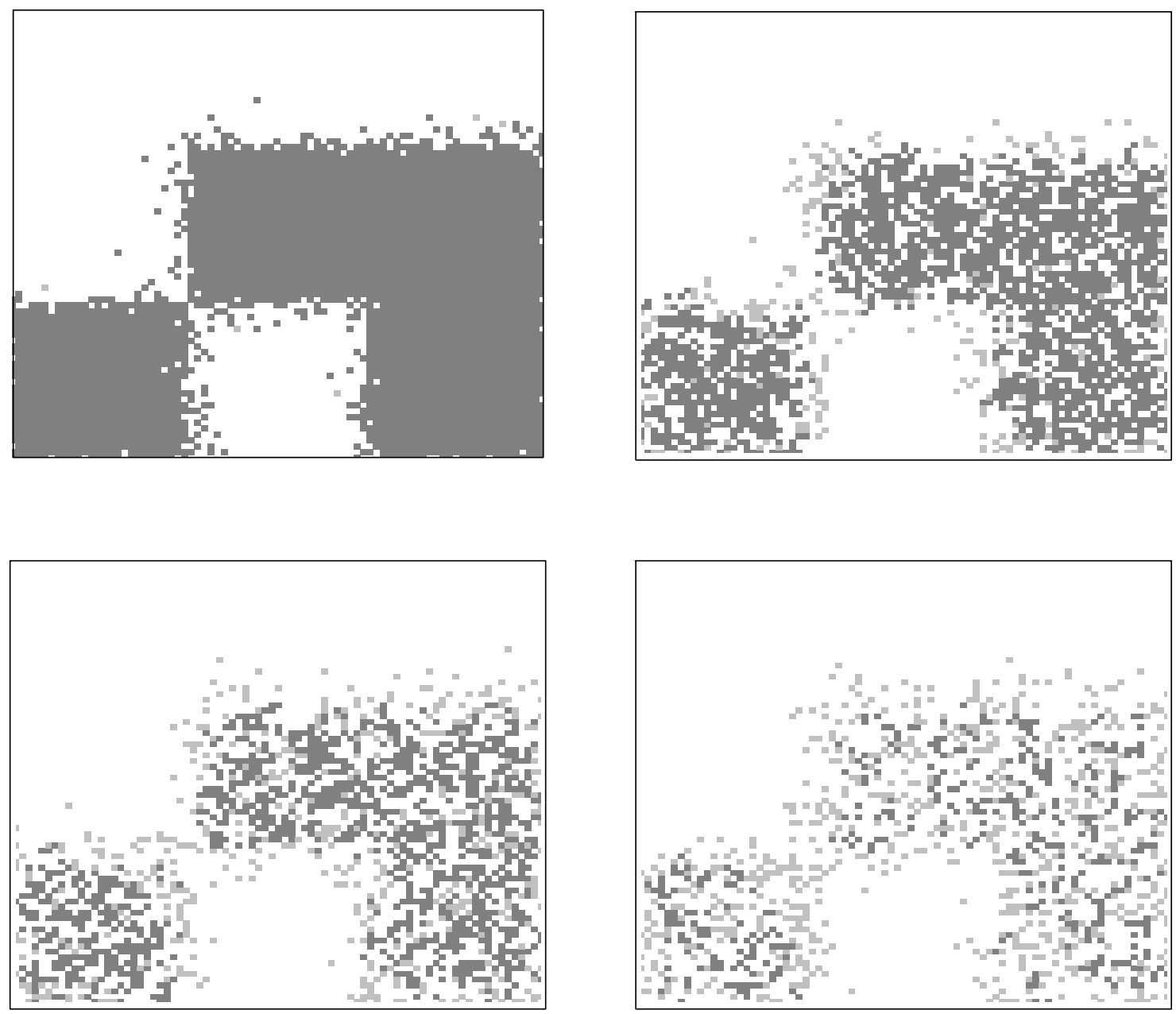

Fig. 5. Horizontal sections through the biofilm, for the same set of probabilities as in Fig. 4 and at time $t=20$ (cf. Fig. 4 - right). In the upper left a section just above the substrate (layer 11) shows the compact base of the four bacterial towers and some aggregation on the periphery. The remaining sections, at layer 19 (top right), 20 (bottom left) and 21 (bottom right), show the fractal character of the tower tops. A hierarchy of bacterial islands (dark) is observable, amidst nutrient (light) and water (white).

long times, long after the bacterial towers are formed, the remaining waterdispersed nutrient particles can be considered as independent walkers - an assumption verified by keeping track of the asymptotic monomer character of nutrient matter in the simulations - and thus the asymptotics is again governed by $d M \propto-M d t$, implying exponential decay.

Just like in the previous simulations without diffusion, a carrying capacity distribution is found, which is controlled by the initial stochastic deposition of nutrient pillars. For a given number of nutrient pillars, the carrying capacity $K$ 

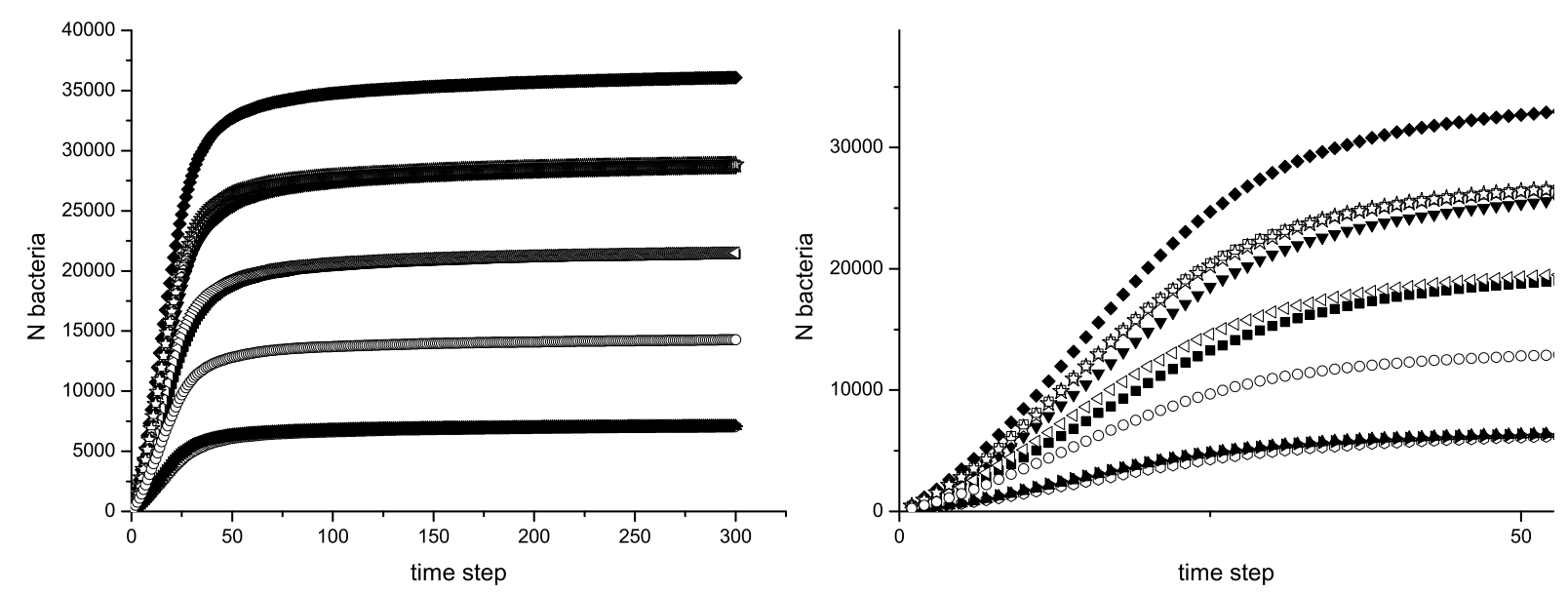

Fig. 6. The number of bacterial cells versus time for growth probability $G=0.2$ and diffusion probability $I=0.2$, for 10 samples randomly drawn with $P=0.33$. The different curves, each with its own carrying capacity $N(\infty)=K$, correspond to different numbers of randomly deposited nutrient pillars (each pillar eventually leads to 7290 bacterial "cells"). In the presence of diffusion the fluctuations of $N(t)$, given a fixed number of nutrient pillars and thus given $K$, are not small, so curves bunch into bands rather than into a sharp line. On the left the full evolution is shown on a time scale long enough to observe saturation, while on the right the detail for a shorter time scale is presented, just long enough to form the towers.

is fixed. To illustrate how this $K$-distribution emerges we present a histogram (Fig.7) giving the frequency of occurrence of $N(10)$, the population after ten time steps, for 1000 samples with $P=0.33, G=0.8$ and $I=0.8$. Note the fine structure of the main peaks. To optimize the resolution we have excluded from the viewing window the trivial peak at $N=0$ (no nutrient was put) and a small but not negligible eighth peak (outside of view, to the right). Note that, as time progresses, these peaks sharpen and converge to a simple set of ten $K$-values corresponding to a population filling all nutrient cells originally contained in the pillars. This sharpening is particular to our simple model, and will no longer occur when additional realistic ingredients such as antibiotics, detachment of bacteria and rinsing water flow is allowed, causing fluctuations of $N(\infty)$.

\section{Comparison with the hierarchical population model}

The random hierarchical population model [6] generates bacterial towers through a more macroscopic construction algorithm. A typical local population $N(x, y ; t)$ grown over a substrate in the $x y$-plane, for $t=4$ (after four generations) and for $P=0.33$ is shown in Fig.8.

A vertical (left) and a horizontal (right) section through the density $N(x, y)$ of 


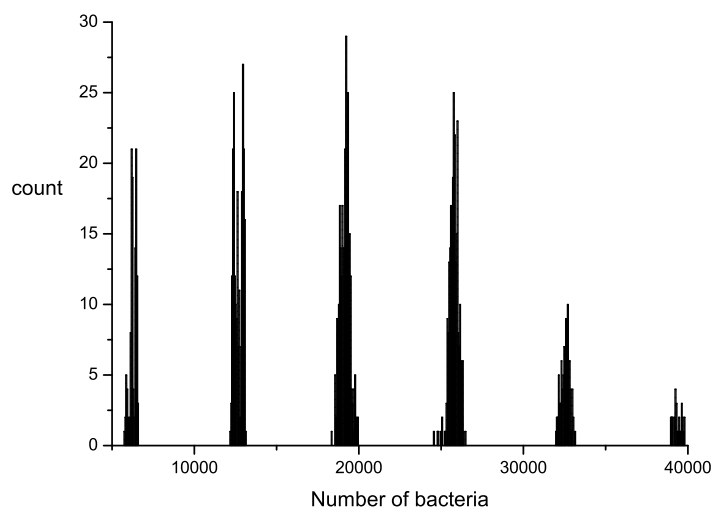

Fig. 7. Population distribution over 1000 samples taken at time step $t=10$, for $P=0.33, G=0.8$ and $I=0.8$, reflecting the "density-of-states" of the bands of bunching $N(t)$-curves at that particular time.

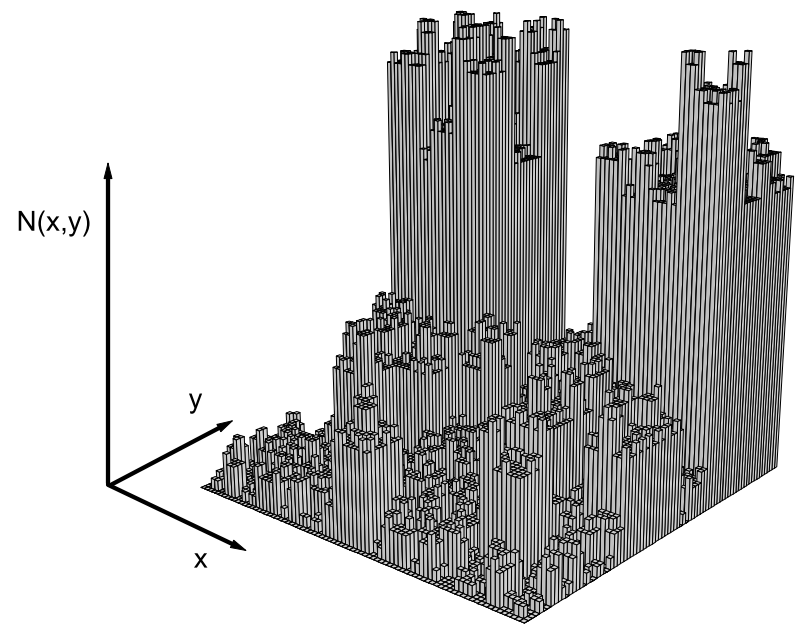

Fig. 8. Bacterial population density from the hierarchical model, consisting of two "towers" and similar replicas on smaller length scales. Note that not height but density $N$ is plotted, which is equivalent to a compactification or compression of the real-space three-dimensional biofilm against the substrate.

Fig.8 are shown in Fig.9. Obviously, bacterial colonies of the type depicted in Fig.4 (right) and in Fig.5 are fairly well represented by Figs.8-9. The differences reside in the details of the self-similar properties intrinsic to the asymptotic long-time behavior of the hierarchical model and not to that of the simulations.

Typical population curves $N(t)$ produced in the hierarchical model are displayed in Fig.10, for $P=0.33$ and length rescaling parameter $\lambda=3$. After a short transient, the populations saturate exponentially rapidly to their carrying capacity. For long times, self-similar carrying capacity distributions result [6], which bear a resemblance to that of Fig.7, except in the details of the fractal geometry. We now turn to the important question of whether we can 

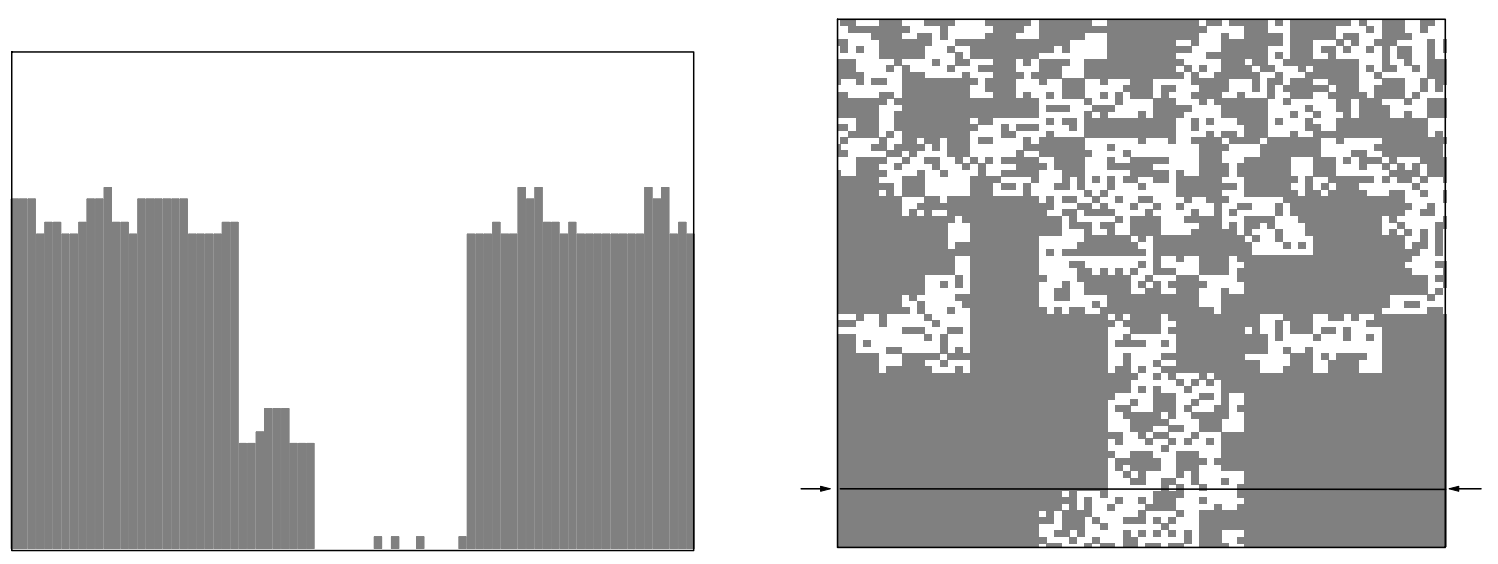

Fig. 9. Vertical (left) and horizontal (right) sections through the density plot of Fig.8. The horizontal section is taken at the bottom of the density "landscape". The vertical section is taken perpendicular to the line indicated in the horizontal section. The figures are rotated by $180^{\circ}$ in the $x y$-plane relative to Fig. 8 .

provide a microscopic basis for the rescaling factor $\lambda$.

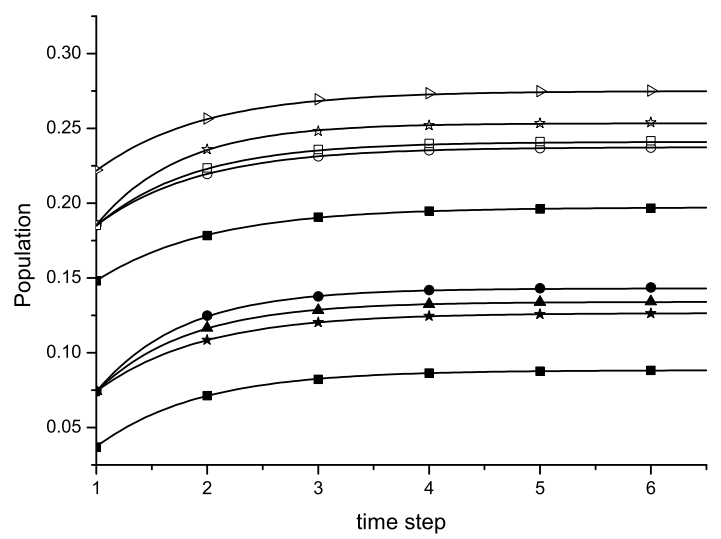

Fig. 10. Hierarchical-model population curves for 9 samples generated with $P=0.33$. Fast exponential convergence to the respective carrying capacities is observed, the time scale of which is set through a heuristic length rescaling factor $\lambda$ (here, $\lambda=3$ ). The population corresponds to the integral over the density $N(x, y)$ defined over the unit square, and the time step is in units of generations. The curves through the data are guides to the eye.

In the exactly soluble random hierarchical population model the average excess population of bacteria $\bar{N}(t)$ obeys the simple time evolution in discrete time $t=n[6]$,

$$
\bar{N}(n)=\left(1-\lambda^{-n}\right) \bar{K},
$$


where $\bar{K}$ denotes the quenched average carrying capacity. This quantity is proportional to the birth probability $B$ (in the present context the probability of death, $D$, equals zero, because no antibiotic or other bactericidal agent is considered)[6]. Therefore, $\bar{K} \propto B$. The exponential saturation is characterized by a rescaling factor $\lambda$, which is introduced ad hoc in that model to incorporate various possible conditions leading to a convergent population (limited nutrient, temperature boost or quench, diffusion without convection, bacterial motility, etc.). The asymptotic behavior of $\bar{N}$ is conspicuous by rewriting (4) in the form

$$
\bar{N}(t)=\left(1-e^{-(\log \lambda) t}\right) \bar{K}
$$

In our CA simulations without diffusion, the towers fill up completely and compactly with bacteria (eventually without surface roughening), and the average carrying capacity is proportional to $P$, the nutrient pillar deposition probability. Precisely the same asymptotic result can be achieved in the hierarchical population model, provided we set $B=P$ in the first generation, and $B=1$ in all subsequent generations, while restricting the growth to the plaquettes where towers were grown in generation 1 (i.e., setting $B=0$ for substrate areas where no nutrient was put). Clearly, the correspondence between $\lambda$ in that model and the variables of our present simulation is

$$
\log \lambda=1 / \tau \propto G
$$

This convergence rate applies not only to the average but also to each curve of Fig.10 individually, since the process is self-averaging for long times [6]. From (6) we learn that a constant microscopic ("cellular") growth probability $G$ combined with a finite nutrient supply, fixing $K$, leads to an exponential saturation, justifying the use of and providing a microscopic interpretation for the constant rescaling factor $\lambda$ in the hierarchical population model.

Including nutrient diffusion in the simulations affects the asymptotic longtime behavior in that a non-zero nutrient/water interchange probability $I$ strongly increases the saturation time. Further, rough biofilm surfaces result. The hierarchical model is ideally suited to generate similar roughness, with marginal fractal geometry, in the spatial distribution of the population density $N(x, y ; t)$ (Fig.8). Note that this density does not provide full information about the three-dimensional biofilm structure - in contrast with the simulations which give the full 3D bacterial "cell" population $N(x, y, z ; t)$ and nutrient "cell" mass $M(x, y, z ; t)$. The hierarchical-model population density yields a compactification in the form of a single-valued density or height profile defined over a 2D support, the substrate. Thus, Fig. 8 shows a relevant population landscape from the hierarchical model, conform with the present 3D simulations including diffusion. Once again, the length rescaling factor $\lambda$ 
can be interpreted using the more microscopic parameters $G$ and $I$ of the CA simulations. Our simulations so far suggest that (6) remains valid for $I \neq 0$.

\section{Conclusions and perspectives}

We have proposed a microscopic cellular automaton describing bacterial tower development on a planar substrate in a strongly non-uniform nutrient environment. The "cells" contain bacteria, nutrient or water. A growth probability $G$ governs the stochastic chemotactic consumption of nutrient cells by adjacent bacterial cells, causing the former to be replaced by bacteria. A probability $I$ controls the interchange of neighboring nutrient and water cells, provoking nutrient diffusion. Nutrient is deposited only once, at the beginning, in the form of drops or pillars, with probability $P$. The strongly non-uniform nutrient supply causes bacterial colonies to grow into towers, and the absence of nutrient refuelling induces exponential saturation of the bacterial population towards a carrying capacity $K$ which depends on $P$. In view of the quenched random deposition a $K$-distribution is found, rather than a single final population.

Typical sigmoid curves are obtained for the time-dependence of the bacterial population. The asymptotic behavior for long times can be mapped onto similar saturation behavior displayed in the hierarchical population model. The phenomenological length rescaling factor in that model, necessary to render a convergent population, can be justified at a more microscopic level through its correspondence to $G$ (and $I$ ) found in the present work. This finding lends support to the applicability of that model as a phenomenological tool for generating fractal towering-pillar biofilm structure.

The simulations can be improved easily by adding new features while keeping maximal simplicity. We consider especially the possibility to add some antibiotic, deposited with probability $Q$, which is capable of killing bacterial cells (with extinction probability $E$ ), and to look for its bactericidal efficiency. In this process an adjacent antibiotic/bacteria pair of cells gets replaced by a pair water/dead-cell. Further, bacterial motility can be included through an additional water/bacteria cell interchange probability, allowing bacterial cells to detach. We have implemented these ideas in a simulation in which on each plaquette nutrient is deposited with $P=0.33$, antibiotics is deposited with $Q=0.33$ and nothing is put with probability $1-(P+Q)$. In a first approximation only dead cells are allowed to detach. All diffusive interchange probabilities (water/nutrient, water/dead-cell, and water/antibiotic) are set to $I=0.6$. Further, $G=0.8$ and $E=1$. Fig. 11 shows a horizontal section through the resulting biofilm structure after about 20 time steps. Interestingly, a bacterial tower has arisen in a nutrient-rich region (right), but only a rel-

atively small hole resulted in the neighbouring antibiotic-rich area (middle). 
The mechanism which prevents massive extinction is the formation of a layer of dead cells mixed with water, separating the lower-lying bacteria from the bactericidal agent. This feature has no counterpart in the hierarchical population model, which is symmetric with respect to interchange of "birth" and "death", but may be instrumental in biofilm resistance to antibiotics.

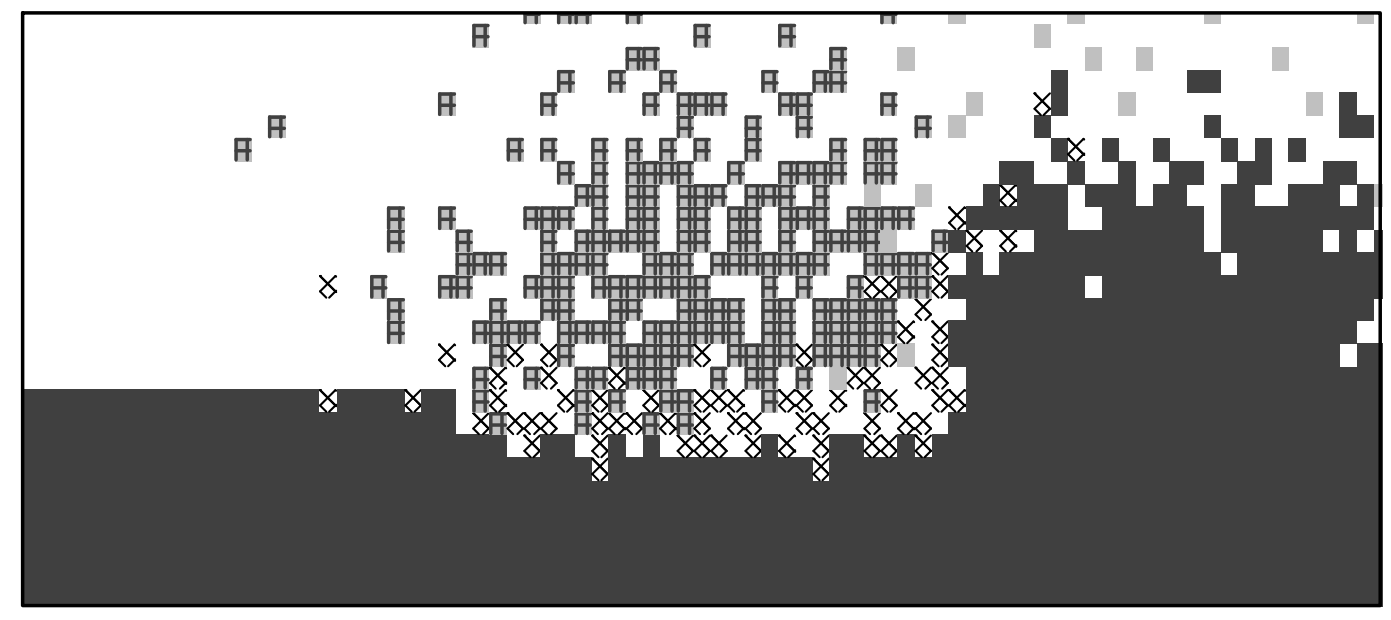

Fig. 11. Vertical section through a simulated 3D "towering-pillar biofilm" consisting of water cells (white), bacterial cells (black), dead bacterial cells (x), nutrient cells (grey) and antibiotic cells(+). Diffusion is allowed for nutrient, antibiotic and dead cells.

In a next step, "rinsing" can be added, entailing removal of detached bacteria, antibiotics and/or nutrient by water flow. Bacterial division in low-nutrient surroundings can also be allowed for, decoupling reproduction from chemotaxis, etc. We stress that the avenues explored here, as well as in the hierarchical model, provide a non-uniform (random) environment for the growth of towering pillar and mushroom structures. We believe that these morphotypes are difficult to understand - and indeed do not emerge easily - in uniform environments assumed when using standard Eden or DLA type aggregation rules. Incidentally, we checked that our approach reproduces pure DLA patterns in the limit of infinite nutrient diffusion (or turbulent convection), randomizing the nutrient mass over space.

\section{Acknowledgments.}

This research was supported by the Flemish Programme FWO-G.0222.02 "Physical and interdisciplinary applications of novel fractal structures". We thank Katarzyna Sznajd-Weron and Jan Żebrowski for useful discussions.

\section{References}

[1] S.W. Hermanowicz, U. Schindler and P. Wilderer, Wat. Sci. Tech. 32, 99 (1995); S.W. Hermanowicz, Wat. Sci. Tech. 39, 107 (1999), and Mathematical 
Biosciences 169, 1 (2001). For a forthcoming overview, see S.W. Hermanowicz, "Biofilm structure: an interplay of models and experiments", in S. Würtz, P.A. Wilderer, P.L. Bishop (Eds.) "Biofilms in wastewater treatment: an interdisciplinary approach", International Water Association Publishing, ISBN: 1843390078.

[2] M. Eden, "A Two-Dimensional Growth Process", 4th Berkeley Symposium, University California Press, 223 (1961).

[3] T.A. Witten and L.M. Sander, Phys. Rev. Lett. 47, 1400 (1981).

[4] S. Wolfram, Rev. Mod. Phys. 55, 601 (1983).

[5] J.D. Murray, "Mathematical Biology", Springer, 1993

[6] J.O. Indekeu and K. Sznajd-Weron, "Hierarchical population model with a carrying capacity distribution for bacterial biofilms", Physical Review E, to appear; physics/0212001 